\title{
Early versus Delayed Complete Lymphadenectomy in Melanoma: Insight from MSLT I
}

\author{
Glenda G. Callender, MD and Kelly M. McMasters, MD, PhD \\ Department of Surgery, James Graham Brown Cancer Center, University of Louisville School of Medicine, Louisville, KY
}

In the December 2010 issue of Annals of Surgical Oncology, Faries et al. present their results from the first Multicenter Selective Lymphadenectomy Trial (MSLT I) regarding morbidity and length of stay for patients who underwent early versus delayed complete lymph node dissection (CLND) for melanoma. ${ }^{1}$ In MSLT I, 937 patients with primary melanoma were randomized to wide local excision (WLE) with sentinel lymph node (SLN) biopsy; early CLND was performed if lymph node metastasis was detected at the time of SLN biopsy. A total of 800 patients were randomized to WLE alone with postoperative observation of nodal basins; delayed CLND was performed if lymph node metastasis was detected by clinical examination during follow-up. Early CLND was performed in 225 patients, and at approximately 5 years of follow-up, 143 patients randomized to WLE alone have undergone delayed CLND (132 with complete data for analysis). Overall, the delayed CLND group demonstrated a statistically significant increase in rate of lymphedema compared with the early CLND group (20.4\% vs. $12.4 \%$; $P=0.04)$. In addition, there was a statistically significant increase in length of hospital stay for patients in the delayed CLND group versus the early CLND group (9.9 vs. 8.3 days; $P=0.021$ ). This finding was hypothesized to result from a higher rate of superficial and deep inguinal lymph node dissection in the delayed CLND group (78\%) compared with the early CLND group (42\%) in which superficial inguinal dissection alone was more common; patients who underwent superficial and deep groin dissection had a longer length of stay compared with

(C) Society of Surgical Oncology 2010

Published Online: 16 December 2010

K. M. McMasters, MD, PhD

e-mail: kelly.mcmasters@louisville.edu superficial groin dissection alone (13.9 vs. 10.2 days; $P=0.009$ ).

This study comes at a time when "less is more" in surgery. We push the envelope with ever more minimally invasive procedures. We consider risks, costs, quality of life, and futility; we often choose to observe where we used to intervene. Standard management of patients with primary melanoma includes SLN biopsy to stage regional lymph node basins, and early CLND for patients with SLNpositive disease. However, CLND carries risks of morbidity, which may substantially alter a patient's quality of life: acute problems with wound healing, and chronic issues, such as weakness, dysesthesia, and lymphedema. Largely because of the associated morbidity of CLND, there exists a growing trend to delay CLND in SLN-positive melanoma. Several recent studies have reported that the actual rate of early CLND in patients with SLN-positive melanoma in the United States is only $50-69 \%$. $^{2,3}$ Patient refusal of the procedure has been cited as the most common reason that patients do not undergo early CLND, but hesitance on the part of many surgeons is probably equally important. ${ }^{4,5}$

However, Faries et al. present an argument for doing more and not less. They argue for early CLND in SLNpositive patients instead of waiting for palpable disease to develop in unstaged regional lymph node basins. Their argument is basically founded on the principle that less morbidity for more patients is better than greater morbidity for fewer patients. It is a difficult concept to quantify, but the data reported in this study add another dimension to the rationale for early CLND in SLN-positive melanoma.

There are two main reasons for performing CLND in SLN-positive melanoma: regional control and cure. It is well-established that CLND for SLN-positive melanoma results in excellent regional disease control. In MSLT I, regional lymph node recurrence after CLND occurred in $4.2 \%$ of patients; similarly, in the Sunbelt Melanoma Trial, 
$4.9 \%$ of patients who underwent CLND developed recurrence in the same nodal basin (unpublished data). ${ }^{6}$ This is clearly superior to $17.9 \%(143 / 800)$ of MSLT I patients who developed clinically detectable regional lymph node metastasis after initially undergoing observation alone. ${ }^{1}$ Regional disease control is a worthy goal in and of itself; this is evident to anyone who has ever managed a patient with bulky, weeping, bleeding tumor replacing a lymph node basin.

But why not wait until a patient develops clinically detectable disease before performing a procedure that has such substantial morbidity? After all, in MSLT I, $84 \%$ of patients who underwent early CLND had no positive nonsentinel lymph nodes, and therefore did not benefit from the procedure. ${ }^{6}$ Faries et al. demonstrated that the morbidity of CLND is greater for patients who develop palpable disease as opposed to patients who undergo early CLND for the microscopic disease detected by SLN biopsy. It should be recognized that these patients were under close observation in the context of a randomized trial and presumably their nodal basin recurrences were detected as early as feasible; patient follow-up and surveillance may not always be as rigorous outside the context of a clinical trial. The authors hypothesize that the increased incidence of lymphedema in patients who develop clinically palpable disease before CLND may result from dysfunction of lymphatics due to nodal involvement by tumor, and/or from the greater extent of surgery and resulting destruction of potential collateral lymphatic channels when CLND is performed for macroscopic instead of microscopic disease. Regardless of the mechanism, these data mean that an already relatively morbid procedure is made even worse. The length of stay data also point to greater morbidity of delayed CLND, although the length of stay in general is longer than one would expect in the United States for CLND; most of these patients were treated in other countries where the policies for hospitalization are different.

Sabel et al. reported similar findings in a retrospective review of 132 patients who underwent inguinal CLND for SLN-positive melanoma versus 80 patients who underwent inguinal CLND for clinically palpable disease. ${ }^{7}$ Wound complications occurred in a statistically significantly higher percent of patients with palpable disease compared with patients with SLN-positive disease (28\% vs. $14 \%$; $P=0.02$ ). The authors concluded that the benefit of performing a staging SLN biopsy in melanoma is that patients can undergo early CLND, thus preventing the increased morbidity of delayed inguinal CLND for palpable disease.

Another argument for early CLND is the potential for cure. We do not know whether CLND after positive SLN biopsy confers a survival advantage. The number of patients with melanoma who are expected to be cured by
CLND is relatively small: only approximately $16 \%$ of patients who undergo SLN biopsy are expected to have a positive sentinel node, and of those, only approximately 8-33\% have other positive nonsentinel nodes that are removed during CLND (16\% in MSLT I) ${ }^{6,8}$ The unknown variable is how many of those patients will never develop distant metastasis and therefore are cured with CLND. Subgroup analysis of MSLT I suggests that SLN-positive patients who underwent CLND had improved survival compared with those who developed palpable recurrence in the WLE-only group. Subgroup analysis, however, is always subject to criticism and is not definitive. Therefore, MSLT II was initiated in 2004 in an attempt to answer the question of whether there exists a survival benefit for CLND. In MSLT II, patients already known to have SLNpositive melanoma are randomized to immediate CLND or close observation of lymph node basins using ultrasound surveillance.

In summary, until conclusive evidence is reached, patients with primary cutaneous melanoma should undergo WLE with SLN biopsy to stage regional lymph node basins. SLN-positive melanoma should ideally be managed by enrollment into MSLT II. Certainly some patients will choose not to participate in such a trial, and some patients do not have access to such a trial; in these patients, management strategies may be subject to debate. Indeed there are some circumstances in which CLND may not be appropriate, such as an older patient with considerable medical comorbidity who might reasonably be expected to die of other causes before dying of melanoma-related disease. However, the results of the study by Faries et al., along with other data, strongly suggest that CLND should remain standard practice for the overwhelming majority of SLN-positive patients.

\section{REFERENCES}

1. Faries MB, Thompson JF, Cochran A, Elashoff R, Glass EC, Mozzillo N, et al. The impact on morbidity and length of stay of early versus delayed complete lymphadenectomy in melanoma: results of the Multicenter Selective Lymphadenectomy Trial (I). Ann Surg Oncol. 2010;17:3324-29.

2. Bilimoria KY, Balch CM, Bentrem DJ, Talamonti MS, Ko CY, Lange JR, et al. Complete lymph node dissection for sentinel nodepositive melanoma: assessment of practice patterns in the United States. Ann Surg Oncol. 2008;15:1566-76.

3. Cormier JN, Xing Y, Ding M, Lee JE, Mansfield PF, Gershenwald $\mathrm{JE}$, et al. Population-based assessment of surgical treatment trends for patients with melanoma in the era of sentinel lymph node biopsy. J Clin Oncol. 2005;23:6054-62.

4. Kingham TP, Panageas KS, Ariyan CE, Busam KJ, Brady MS, Coit DG. Outcome of patients with a positive sentinel lymph node who do not undergo completion lymphadenectomy. Ann Surg Oncol. 2010;17:514-20.

5. McMasters KM. Why does no one want to perform lymph node dissection anymore? Ann Surg Oncol. 2010;17:358-61. 
6. Morton DL, Thompson JF, Cochran AJ, Mozzillo N, Elashoff R, Essner R, et al. Sentinel-node biopsy or nodal observation in melanoma. N Engl J Med. 2006;355:1307-17.

7. Sabel MS, Griffith KA, Arora A, Shargorodsky J, Blazer DG 3rd, Rees $\mathrm{R}$, et al. Inguinal node dissection for melanoma in the era of sentinel lymph node biopsy. Surgery. 2007;141:728-35.
8. Gershenwald JE, Andtbacka RHI, Prieto VG, Johnson MM, Diwan $\mathrm{AH}$, Lee JE, et al. Microscopic tumor burden in sentinel lymph nodes predicts synchronous nonsentinel lymph node involvement in patients with melanoma. J Clin Oncol. 2008;26:4296-303. 